\title{
Effects of Caffeine on Auditory- and Vestibular-Evoked Potentials in Healthy Individuals: A Double-Blind Placebo-Controlled Study
}

\author{
Elham Tavanai, Saeid Farahani, Mansoureh Adel Ghahraman, \\ Saleheh Soleimanian, and Shohreh Jalaie \\ Department of Audiology, School of Rehabilitation, Tehran University of Medical Sciences, Tehran, Iran
}

\author{
Received June 9, 2019 \\ Revised July 25, 2019 \\ Accepted September 16, 2019
}

Address for correspondence

Saeid Farahani, PhD

Department of Audiology,

School of Rehabilitation,

Tehran University of Medical Sciences,

Pich-e-shemiran, Enghelab Avenue,

Tehran 009821, Iran

Tel +98-21-77530636

Fax +98-21-77684889

E-mail s_farahani@tums.ac.ir
Background and Objectives:The blockage of adenosine receptors by caffeine changes the levels of neurotransmitters. These receptors are present in all parts of the body, including the auditory and vestibular systems. This study aimed to evaluate the effect of caffeine on evoked potentials using auditory brainstem responses (ABRs) and cervical vestibular-evoked myogenic potentials (cVEMPs) in a double-blind placebo-controlled study. Subjects and Methods: Forty individuals (20 females and 20 males; aged 18-25 years) were randomly assigned to two groups: the test group (consuming $3 \mathrm{mg} / \mathrm{kg}$ pure caffeine powder with little sugar and dry milk in $100 \mathrm{~mL}$ of water), and the placebo group (consuming only sugar and dry milk in $100 \mathrm{~mL}$ water as placebo). The cVEMPs and ABRs were recorded before and after caffeine or placebo intake. Results: A significant difference was observed in the absolute latencies of I and III $(p<0.010)$, and $V(p<0.001)$ and in the inter-peak latencies of III-V and I-V $(p<0.001)$ of ABRs wave. In contrast, no significant difference was found in cVEMP parameters (P13 and N23 latency, threshold, P13-N23 amplitude, and amplitude ratio). The mean amplitudes of P13-N23 showed an increase after caffeine ingestion. However, this was not significant compared with the placebo group ( $p>0.050)$. Conclusions: It seems that the extent of caffeine's effects varies for differently evoked potentials. Latency reduction in ABRs indicates that caffeine improves transmission in the central brain auditory pathways. However, different effects of caffeine on auditory- and vestibular-evoked potentials could be attributed to the differences in sensitivities of the ABR and cVEMP tests.

J Audiol Otol 2020;24(1):10-16

KEY WORDS: Caffeine $\cdot$ Adenosine $\cdot$ Auditory brain stem responses Cervical vestibular evoked myogenic potentials.

\section{Introduction}

Caffeine (1,3,7-trimethylxanthine) is believed to be the most widely and frequently consumed psychoactive substance in the world and is a natural constituent of numerous available foods and beverages, such as coffee, tea, cocoa products, and cola products [1]. Various hypotheses have been postulated for the mechanisms of action of caffeine, includ-

This is an Open Access article distributed under the terms of the Creative Commons Attribution Non-Commercial License (https://creativecommons.org/licenses/by-nc/4.0/) which permits unrestricted non-commercial use, distribution, and reproduction in any medium, provided the original work is properly cited. ing blocking of adenosine receptors, mobilization of intracellular calcium, inhibition of phosphodiesterases, and binding of caffeine to benzodiazepine receptors [2]. However, the chief effect of caffeine is the blocking of adenosine receptors $[3,4]$. These properties allow caffeine to affect many human tissues, including those of the central nervous system, cardiovascular system, and both smooth and skeletal muscular systems $[4,5]$. Additionally, several studies have suggested that some effects of caffeine could be due to its effects on formation and release of neurotransmitters [2], for example, levels of neurotransmitters such as glutamate, serotonin, noradrenaline, acetylcholine, and dopamine change due to adenosine 
blockage $[1,4]$. Adenosine not only acts as a neurotransmitter and neuromodulator, but is also a constituent of other important bioactive molecules including adenosine triphosphate, ribonucleic acid, and secondary messengers such as cyclic adenosine monophosphate [4].

There is extensive literature examining the effects of caffeine on physiological, electrophysiological, and cognitive functions, and it is believed to influence mood and cognitive performance $[1,3,4]$. However, there is limited research focusing on the effects of caffeine on the auditory and especially vestibular system. Previous studies have evaluated effects of caffeine on the organ of corti [6], auditory-evoked potentials [710], and vestibular system [11-16]. They showed that caffeine significantly suppressed the compound action potential of the auditory nerve and summation potential at low intensity, and increased the N1 latency at high and low intensities [6]. Additionally, caffeine reduced the distortion product otoacoustic emissions at low intensities and increased it at high intensities, leading to the shortening of the outer hair cells [6].

In auditory brain stem evoked responses (ABRs), caffeine ingestion significantly reduced the absolute and inter-peak latencies [7,10] and increased the amplitude of wave V [7]. However, there are conflicting results from numerous studies with respect to upper level potentials. In different studies, caffeine invoked reduced P1 middle latency response [7], reduced P300 latency and amplitude [8], and increased P1, P2, and P3b amplitude without any effect on the latency [9].

There are also few studies on the effect of caffeine on the vestibular system. There were no significant effects found in studies performed for tests of caloric $[11,12]$, posturography [13], rotary chair [14], and vestibular-evoked myogenic potentials (cVEMP) $[12,15,16]$. However, studies wherein oculomotor tests were performed showed that caffeine treatment significantly reduced the saccadic eye movements of smooth pursuit in schizophrenic patients [17]. McNerney, et al. [14] also reported statistically significant differences in the results of several oculomotor tests after caffeine ingestion, including vertical saccades, horizontal saccades, and optokinetics. Furthermore, Pilli, et al. [18] reported that caffeine could reduce the saccade latency significantly.

It has been hypothesized that caffeine causes an increase in the release of neurotransmitters, such as glutamate, by interacting with the adenosine receptors, thus improving the sensory perception of auditory stimuli after caffeine intake [7]. This is important because adenosine is a constitutive metabolite of all cells and is present in both the auditory [19] and vestibular systems [20].

However, the effects of caffeine on auditory and vestibular systems is still under debate, and there are no placebo-based clinical trials evaluating its effect on these systems and specially evoked potentials. Therefore, the main purpose of this study was to evaluate the effects of caffeine on auditory- and vestibular-evoked potentials in a randomized, double blind, placebo-controlled trial. Since caffeine affects the central nervous system as well as neuromuscular function, and cVEMP is result of the coordination between sensory and neuromuscular functions, we aimed to assess the effects of caffeine on cVEMP and ABRs.

\section{Subjects and Methods}

The present study was designed as a randomized, double blind, placebo-controlled, interventional study approved by the Ethics Committee of Tehran University of Medical Sciences with code of BP-QP-110-01.

\section{Participants}

The study group recruited 40 individuals (20 males, 20 females) aged 18-25 years from the Rehabilitation Faculty of Tehran University of Medical Sciences, Tehran, Iran. Individuals with no history of neurologic, myogenic, balance and cervical disorders, ear diseases, psychiatric illness, and habitual smoking and drinking, as well as those consuming vestibulotoxic drugs, such as gentamycin and neomycin, were included. Additionally, to eliminate the effect of weight, body mass index of $18.5-24.9 \mathrm{~kg} / \mathrm{m}^{2}$ was considered as an inclusion criterion. Moreover, individuals with low caffeine intake ( $<200 \mathrm{mg}$ /day of caffeine-containing substances) were eligible to participate in this study.

All participants were asked to abstain from caffeine-containing substances (tea, coffee, and cola) for at least 6 hours before the test. Informed consent was obtained from all participants. To assure the health of the auditory system, participants underwent otoscopy (Reister, Jungingen, Germany), immittance audiometry (Zodiac, Madsen Co., Taastrup, Denmark), and pure tone audiometry (AC40, Interacoustics Co., Middelfart, Denmark) examinations. The inclusion criteria were normal tympanogram (static compliance between $0.3-1.6$, middle ear pressure between $-100-+50$ ), acoustic reflexes with thresholds between 70-100 decibels in hearing level (dB $\mathrm{HL}$ ) in immittance audiometry, and air conduction and bone conduction thresholds of $\leq 15 \mathrm{~dB}$ in pure tone audiometry.

\section{Experimental procedure}

Initially, the ABRs and cVEMP were recorded in all participants by the experimenter. The participants were then randomly assigned to one of two groups, and both participants and experimenter were blind to the group allotment. The caf- 
feine and placebo allotment was written in 40 similar envelopes, and $3 \mathrm{mg} / \mathrm{kg}$ caffeine $(\mathrm{n}=20)$ and $0 \mathrm{mg} / \mathrm{kg}$ caffeine or placebo $(n=20)$ were placed in a container by a research collaborator. The standard dose of caffeine used in most studies is $3 \mathrm{mg} / \mathrm{kg}$. The participants chose one of the envelopes randomly and gave it to the collaborator without opening it, and were thus allotted to one of two groups: test group and placebo group. Following this, the body weights of participants were measured using a weighing scale. The collaborator measured the caffeine amount (Human Pharmaceutical, Roma, Italy) per $\mathrm{kg}$ of body weight using the analyzer research scale and dissolved it in $100 \mathrm{~mL}$ of water. Moreover, a little powdered milk and sugar were added to improve the flavor, and the powdered milk helped achieve similar appearance of the drinks in both groups. The used cups were disposable and non-transparent. The collaborator wrote the name of the participants and the weight of the materials in a separate list not accessible to the participants. Since caffeine reaches its highest concentration in blood plasma within 30-60 min of ingestion, the tests were repeated after about $40 \mathrm{~min}$. In order to eliminate the order effect, the test was conducted randomly once for the right ear and once for the left ear.

\section{Recording procedure}

The cVEMP was recorded with 500 hertz $(\mathrm{Hz})$ tone burst stimuli (2-1-2 duration) using insert earphone, at a repetition rate of $5.1 / \mathrm{s}$, intensity of $95 \mathrm{~dB} \mathrm{HL}, 10-1,500 \mathrm{~Hz}$ band-pass filter, 100 stimuli, 5000X gain, and with the active surface electrode placed over the upper one-third of the sternocleidomastoid muscle, reference electrode over the upper sternum, and ground electrode on the forehead. P13 and N23 latency, P13-N23 amplitude, threshold, and asymmetric ratio (AR) parameters were recorded. For equal contraction of muscles on both sides, the feedback method was adopted. ABRs were recorded at a repetition rate of $9.1 / \mathrm{s}$, with rarefaction click stimuli, intensity of $90 \mathrm{~dB}$ peSPL (peak sound pressure level), $100-3,000 \mathrm{~Hz}$ filter, with the active surface electrode placed over the stimulated ear, reference electrode on the forehead, and ground electrode over the contralateral ear. The skin electrode contact impedance was maintained at $<5 \mathrm{k} \Omega$.

\section{Statistical analysis}

The normality of variables was assessed using the Kolmogorov-Smirnov Goodness-of-Fit test. The significance level was set at $p<0.01$ for ABR and $p<0.05$ for cVEMP. Wilcoxon test and Mann-Whitney U tests were used for within-group and between-group comparisons, respectively.

\section{Results}

This study included 40 individuals of both sexes with a mean age of 23 years. Statistical analysis during the pre-ingestion session revealed that all parameters of cVEMP (P13, N23 latency, P13-N23 amplitude, AR, and threshold) and ABRs (absolute I, III, V, I-III, III-V and I-V inter-peak latency, and V/I amplitude ratio) were homogenous in both groups, and there was no significant difference between the groups for any parameters $(p>0.05)$. There was no significant difference between both ears; hence, their results were combined. The relative changes in parameters of ABRs and cVEMP are presented in Table 1 and 2. Relative changes were calculated as the difference between the pre- and post-data, divided by the standard deviation of pre-data.

In ABRs, a significant reduction was found in the test group compared with placebo group in absolute latencies of I, III, and V and inter-peak latencies of III-V and I-V (Table 1). The relative changes in absolute latencies of I, III, and V and inter-peak latencies of III-V and $\mathrm{I}-\mathrm{V}$ are shown in Table 1 . They increased or remained unchanged in the placebo

Table 1. Relative changes in parameters of auditory brainstem responses in the test group and placebo group

\begin{tabular}{lccc}
\hline \multicolumn{1}{c}{ Parameters } & $\begin{array}{c}3 \mathrm{mg} / \mathrm{kg} \\
\text { caffeine group }\end{array}$ & Control group p value \\
\hline $\begin{array}{l}\text { Absolute latency } \\
\text { I }\end{array}$ & $-0.01(0.041)$ & $0.004(0.01)$ & $0.004^{*}$ \\
III & $-0.006(0.017)$ & $0.002(0.007)$ & $0.002^{*}$ \\
V & $-0.01(0.016)$ & $0.0007(0.008)$ & $<0.001^{\dagger}$ \\
Inter-peak latency & & & \\
I-III & $-0.009(0.084)$ & $-0.004(0.028)$ & 0.215 \\
III-V & $-0.03(0.065)$ & $0.008(0.063)$ & $<0.001^{\dagger}$ \\
I-V & $-0.01(0.041)$ & $0.002(0.013)$ & $<0.001^{\dagger}$ \\
Amplitude ratio & & & \\
V/I & $0.74(5.26)$ & $0.20(2.74)$ & 0.718 \\
\hline
\end{tabular}

Values are presented as mean (standard deviation) unless otherwise indicated. $* p<0.010,{ }^{\dagger} p<0.001$.

Table 2. Relative changes in the parameters of cervical vestibularevoked myogenic potentials in the test group and placebo group

\begin{tabular}{lccc}
\hline \multicolumn{1}{c}{ Parameters } & $\begin{array}{c}3 \mathrm{mg} / \mathrm{kg} \\
\text { caffeine group }\end{array}$ & Control group $\mathrm{p} \mathrm{value}$ \\
\hline Latency & & & \\
P13 & $0.005(0.03)$ & $0.001(0.01)$ & 0.695 \\
N23 & $0.008(0.04)$ & $-0.001(0.02)$ & 0.384 \\
Amplitude & & & \\
P13-N23 & $0.14(0.54)$ & $0.0006(0.46)$ & 0.061 \\
Asymmetric ratio & $-1.33(0.05)$ & $-1.80(0.03)$ & 0.794 \\
Threshold & $-0.014(0.04)$ & $-0.019(0.04)$ & 0.703 \\
\hline
\end{tabular}

Values are presented as mean (standard deviation) unless otherwise indicated. 
group, but decreased in the test group. In the placebo group, the latencies of wave I, III, and V were $2.47 \pm 0.14,4.57 \pm$ 0.13 , and $6.54 \pm 0.20$ milliseconds ( $\mathrm{ms}$ ) before and $2.48 \pm$ $0.14,4.66 \pm 0.14$, and $6.54 \pm 0.20 \mathrm{~ms}$ after the intervention, respectively. In the test group, the latencies of I, III, and V were $2.51 \pm 0.16,4.56 \pm 0.15,6.53 \pm 0.21 \mathrm{~ms}$ before the intervention, respectively, and reduced to $2.46 \pm 0.16,4.53 \pm 0.11$, and $6.45 \pm 0.20 \mathrm{~ms}$, respectively, after caffeine intake. The inter-peak latencies of III $-\mathrm{V}$ and $\mathrm{I}-\mathrm{V}$ in the test group were $1.97 \pm 0.13 \mathrm{~ms}$ and $4.03 \pm 0.17 \mathrm{~ms}$ before the intervention, which decreased to $1.91 \pm 0.15 \mathrm{~ms}$ and $3.97 \pm 0.20 \mathrm{~ms}$, respectively. However, in the placebo group, the latency of III$\mathrm{V}(1.97 \pm 0.14 \mathrm{~ms})$ remained unchanged, while the latency of $\mathrm{I}-\mathrm{V}$ increased slightly (from $4.06 \pm 0.20 \mathrm{~ms}$ to $4.07 \pm 0.20 \mathrm{~ms}$ ).

The latency of I-III did not show a significant difference between the groups, but showed a slight decrease in both groups. The latency of I-III before and after caffeine intake was $2.06 \pm 0.13 \mathrm{~ms}$ and $2.04 \pm 0.13 \mathrm{~ms}$ in the test group, and $2.09 \pm 0.15 \mathrm{~ms}$ and $2.08 \pm 0.13 \mathrm{~ms}$ in the placebo group, respectively. No significant difference was found between the groups for V/I amplitude of ABRs. V/I amplitude of ABRs increased in both groups, from $6.95 \mu \mathrm{v}$ to $12.09 \mu \mathrm{v}$ in the test group and from $5.22 \mu \mathrm{v}$ to $6.30 \mu \mathrm{v}$ in the placebo group.

Fig. 1 and 2 illustrate the relative changes of absolute and inter-peak latencies in both groups. As shown, all absolute latencies of ABRs decreased in the test group and increased in the placebo group. In the test group, maximum decrease was observed in the absolute latencies of wave I and V and interpeak latency of III-V, and minimum decrease was noted in absolute latency of III and inter-peak latency of I-III.

No statistically significant differences were observed in any of the cVEMP parameters in the test group compared with the placebo group $(p>0.05)$. The mean P13-N23 amplitude of cVEMP increased in both groups. However, the difference between the groups was not statistically significant. The only considerable finding was the changes in P13-N23 amplitude that bordered the significance level $(p=0.061)$. The mean P13$\mathrm{N} 23$ amplitude in the test group was $172.02 \pm 103.42 \mu \mathrm{v}$ during pre-ingestion session that increased to $197.74 \pm 101.27 \mu \mathrm{v}$ after caffeine intake. However, the mean P13-N23 amplitude in the placebo group was $137.95 \pm 78.17 \mu \mathrm{v}$ that increased slightly to $138.04 \pm 80.83 \mu \mathrm{v}$.
Fig. 1. The effects of caffeine and placebo on absolute latencies of auditory brain stem responses. Relative changes in the absolute latencies of all auditory brain stem responses waves were significantly greater in caffeine group compared with the placebo group $\left({ }^{*} p<0.010,{ }^{\dagger} p<0.001\right)$.

Fig. 2. The effects of caffeine and placebo on inter-peak latencies of auditory brain stem responses. Relative changes in the inter-peak latencies of III-V and I-V waves of auditory brain stem responses were significantly greater in caffeine group compared with the placebo group. No significant changes was seen in IIII inter-peak latency in caffeine group compared with the placebo group $\left({ }^{\dagger} p<0.001\right)$.
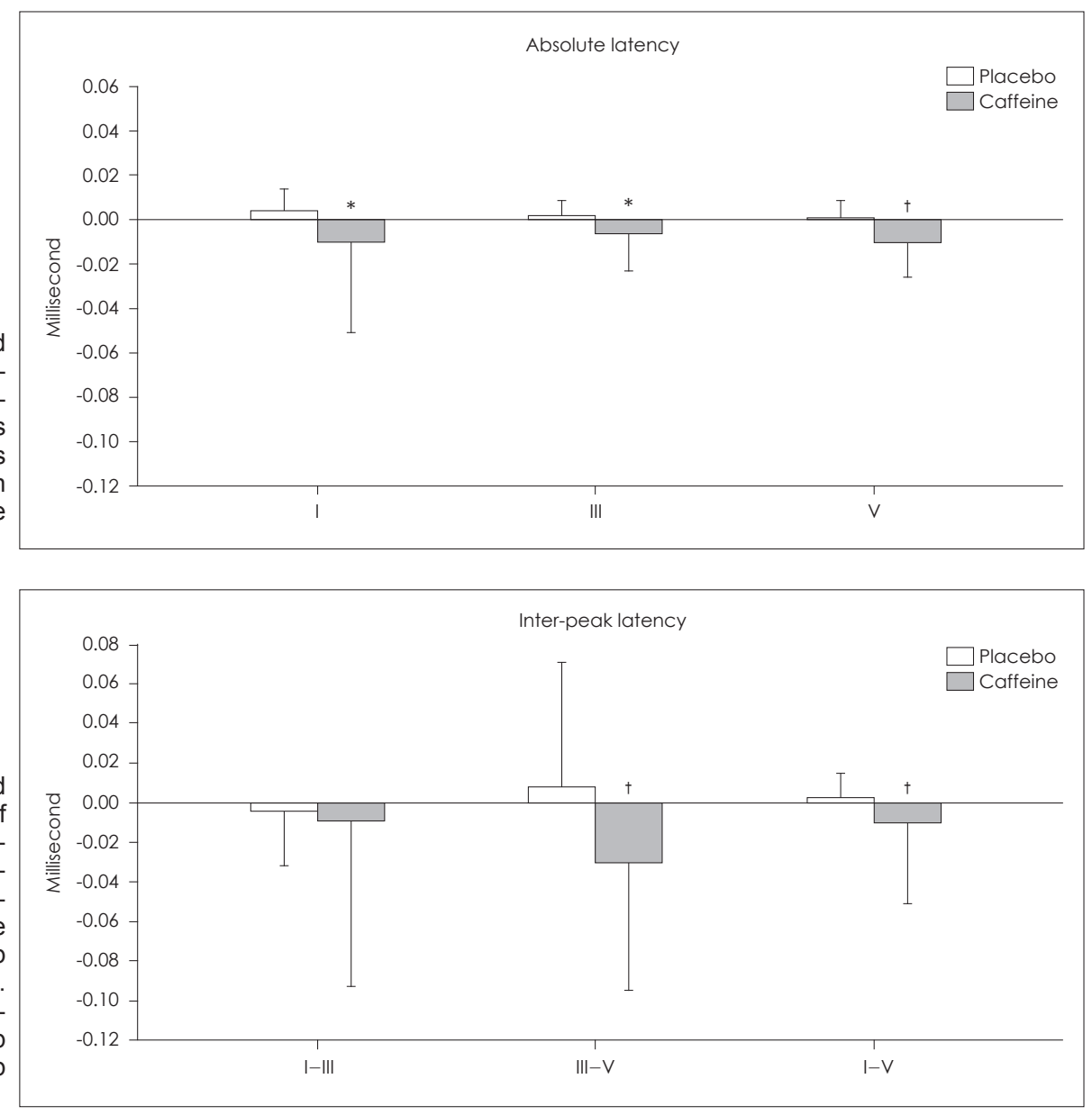
The P13 and N23 latency also increased slightly in the test group after caffeine ingestion. In the test group, the latency of P13 and N23 increased from $16.61 \pm 0.84 \mathrm{~ms}$ and $24.54 \pm$ $1.45 \mathrm{~ms}$ to $16.67 \pm 0.89 \mathrm{~ms}$ and $24.84 \pm 1.38 \mathrm{~ms}$, respectively. In the placebo group, the latency of P13 increased from $16.91 \pm$ $1.69 \mathrm{~ms}$ to $16.92 \pm 1.56 \mathrm{~ms}$, but that of $\mathrm{N} 23$ fell from $24.89 \pm$ $1.41 \mathrm{~ms}$ to $24.85 \pm 1.28 \mathrm{~ms}$. The threshold of cVEMP showed a slight decrease in both groups.

\section{Discussion}

In general, the findings showed a significant reduction in the absolute latencies of I, III, and V and in the inter-peak latencies of III-V and $\mathrm{I}-\mathrm{V}$ of the ABRs. However, there was no significant difference noted in the parameters of cVEMP in the test group compared with the placebo group. There is limited research focusing on the effects of caffeine on ABRs and cVEMP.

Latency reduction results in $\mathrm{ABR}$ in the present study are concurrent with the study by Dixit, et al. [7] and Shalini, et al. [10]. Dixit, et al. [7] observed significant reduction in absolute latencies of waves IV and V along with I-V inter-peak latency of ABRs. Although they found latency reduction in IIII and III-V waves, the changes were not statistically significant. This was probably because of the low sample size in both studies. However, in the study by Shalini, et al. [10], all waves showed absolute and inter-peak latency reduction, but there was no control group. The findings on the latency reduction also concurred with the studies showing that caffeine decreased the reaction time [21-24]. Seidl, et al. [21] found that the reaction time improved in response to target stimuli after administration of a drink containing caffeine.

In other evoked potentials, Deslandes, et al. [25] found P300 latency reduction at the Fz electrode when the participants consumed caffeine. However, Barry, et al. [9] showed that caffeine had no effect on P1, P2, and P3b latency, but a significant increase was observed in the amplitude of P1, P2, and $\mathrm{P} 3 \mathrm{~b}$ at a dose of $250 \mathrm{mg}$. In contrast, no significant increase in V/I amplitude of ABRs was found in the present study. In the study by Dixit, et al. [7,24], a significant increase was detected in wave $\mathrm{V}$ amplitude of ABRs, while in wave I there was no significant increase. Amplitude rise after caffeine intake have been also reported for other evoked potentials such as event-related potentials [26] and visual evoked potentials [27]. In studies using visual-evoked potentials, $3 \mathrm{mg} / \mathrm{kg}$ caffeine increased the amplitude of P2 and n2b [27]. Although in event related potentials studies, other factors such as higher levels of attention and arousal were triggered by caffeine, they were not comparable with subcortical responses such as ABRs. We analyzed amplitude ratio of $\mathrm{V} / \mathrm{I}$ in the present study and not the absolute amplitude of waves, because amplitude is a variable parameter and amplitude ratio is a more suitable parameter than absolute amplitude, which has not been evaluated in other studies.

It is suggested that latency reduction is caused by a boost in information processing speed [27]. Caffeine stimulates the central nervous system initially at the higher-level functions of the brain, including cognition, memory, attention, and concentration, where altering the peripheral motor responses results in ergogenic action. Caffeine acts mainly by blocking the adenosine receptors, which are responsible for the "fine-tuning" of the neuronal communication. The stimulatory effect of caffeine as represented by the shorter latencies could also represent the speed at which the sensory information is being transmitted to their respective cortices of sensory memory, a part of the brain's cognition [10]. Additionally, it has been reported that caffeine can improve the performance in central auditory behavioral tasks [28]. Accordingly, Taghavi, et al. [28] evaluated the effects of short-term caffeine consumption on speech and sound reception in noise using the acceptable noise level test in healthy individuals. Speech perception in noise is one of the central auditory functions that depends on the interaction of sensory and cognitive processing. They found that the individuals tolerated higher levels of speech in noise [28].

In the present study, no statistically significant difference was found in the parameters of cVEMPs in the test group compared with the placebo group. There is not enough information regarding the effects of caffeine on the vestibular system, particularly on cVEMPs. The absence of significant effect of caffeine on cVEMPs in this study is in agreement with previous studies on the effect of caffeine on vestibular system employing different techniques, especially cVEMPs. There were no significant effects reported in the studies wherein tests of caloric [11,12], posturography [13], rotary chair [14], and cVEMP $[12,15,16]$ were performed. de Sousa and Suzuki [15] administered $420 \mathrm{mg}$ of caffeine, and the cVEMP parameters were compared before and after caffeine intake. No statistically significant difference was found in the test results before and after caffeine ingestion. Similarly, in the study by McNerney, et al. [12], 30 young healthy participants were tested with and without the consumption of moderate amount of caffeine before undergoing caloric and cVEMP tests. The results revealed that a moderate amount of caffeine did not have a clinically significant effect on the results of caloric and cVEMP tests in young healthy adults. However, another study reported statistically significant differences in the results of several oculomotor tests after caf- 
feine ingestion [14].

de Sousa and Suzuki [15] suggested that there is probably a negligible influence of adenosine receptors on the sacculocollic pathway, and further studies are necessary to clarify the distribution of these receptors in the peripheral vestibular pathway. Another probable cause is that the cVEMP is not sufficiently sensitive to caffeine effects. There are also differences in the auditory brain stem responses and cVEMP. Perhaps, the difference between the findings of ABRs and cVEMP studies is due to the wider normal range of latency for the cVEMP than ABRs, where ABRs are a completely neurologic response but cVEMP is a neurologic as well as myogenic response. Thus, ABRs could have higher sensitivity to the caffeine-induced changes as compared to cVEMP. It seems that the extent of caffeine effects on the various evoked potentials is presumably different, probably because caffeine has complex psychophysiological roles and there are evident differences in the body responses of individuals to caffeine.

The findings of the present study about the effects of caffeine on auditory and vestibular systems in particular could be affected by the low statistical power and the small sample size. Moreover, because caffeine dose-response curve is like an inverted "U", and it has different effects at different doses, further studies with larger sample size and higher doses are needed to evaluate the effects of caffeine on auditory and vestibular systems in particular.

In conclusion, it seems that the extent of caffeine's effects on various evoked potentials is different owing to the complex psychophysiological roles of caffeine and differences in the body responses of individuals evoked by it. Latency reduction in ABRs indicated that caffeine improved transmission in the central brain auditory pathways. However, different effects of caffeine on the auditory- and vestibular-evoked potentials are probably because of the different sensitivities of ABRs and cVEMP. To understand the effect of higher doses of caffeine on cVEMP and ABRs, further studies with a larger sample size are required.

\section{Conflicts of interest}

The authors have no financial conflicts of interest.

\section{Author Contributions}

Conceptualization: Saeid Farahani and Mansoureh Adel Ghahraman. Data curation: Elham Tavanai and Saleheh Soleimanian. Formal analysis: Shohreh Jalaie and Elham Tavanai. Funding acquisition: Saeid Farahani. Investigation: Elham Tavanai, Saeid Farahani, and Mansoureh Adel Ghahraman. Methodology: Mansoureh Adel Ghahraman, Saeid Farahani, and Elham Tavanai. Project administration: Elham Tavanai and Saleheh Soleimanian. Supervision: Saeid Farahani and Mansoureh Adel Ghahraman. Writing — original draft: Elham Tavanai, Saleheh Soleimanian, and Shohreh Jalaie. Writ- ing — review \& editing: Saeid Farahani, Mansoureh Adel Ghahraman, and Elham Tavanai.

\section{ORCID iDs}

Elham Tavanai https://orcid.org/0000-0002-9844-3111

Saeid Farahani https://orcid.org/0000-0003-2541-283X

Mansoureh Adel Ghahraman

https://orcid.org/0000-0002-7118-6231

Saleheh Soleimanian https://orcid.org/0000-0002-4427-6311

Shohreh Jalaie https://orcid.org/0000-0001-6044-9617

\section{REFERENCES}

1) Ruxton CHS. The impact of caffeine on mood, cognitive function, performance and hydration: a review of benefits and risks. Nutrition Bulletin 2008;33:15-25.

2) Nehlig A, Daval JL, Debry G. Caffeine and the central nervous system: mechanisms of action, biochemical, metabolic and psychostimulant effects. Brain Res Brain Res Rev 1992;17:139-70.

3) Nawrot P, Jordan S, Eastwood J, Rotstein J, Hugenholtz A, Feeley M. Effects of caffeine on human health. Food Addit Contam 2003;20:130.

4) Martin PY, Hamilton VE, McKimmie BM, Terry DJ, Martin R. effects of caffeine on persuasion and attitude change: the role of secondary tasks in manipulating systematic message processing. Eur J Soc Psychol 2007;37:320-38.

5) Tarnopolsky MA. Effect of caffeine on the neuromuscular system-potential as an ergogenic aid. Appl Physiol Nutr Metab 2008;33: $1284-9$.

6) Bobbin RP. Caffeine and ryanodine demonstrate a role for the ryanodine receptor in the organ of Corti. Hear Res 2002;174:172-82.

7) Dixit A, Vaney N, TandonOP. Effect of caffeine on central auditory pathways: an evoked potential study. Hear Res 2006;220:61-6.

8) Pan J, Takeshita T, Morimoto K. Acute caffeine effect on repeatedly measured P300. Environl Health Prev Med 2000;5:13-7.

9) Barry RJ, Johnstone SJ, Clarke AR, Rushby JA, Brown CR, McKenzie DN. Caffeine effects on ERPs and performance in an auditory Go/NoGo task. Clin Neurophysiol 2007;118:2692-9.

10) Shalini S, Chandra Selvi E, Sai Kumar P. Effect of caffeine on evoked potentials. IOSR J Dent Med Sci 2012;2:43-8.

11) Felipe L, Simões LC, Gonçalves DU, Mancini PC. Evaluation of the caffeine effect in the vestibular test. Braz J Otorhinolaryngol 2005; 71:758-62.

12) McNerney K, Coad ML, Burkard R. The influence of caffeine on calorics and cervical vestibular evoked myogenic potentials (cVEMPs). J Am Acad Audiol 2014;25:261-7.

13) Enriquez A, Sklaar J, Viirre E, Chase B. Effects of caffeine on postural stability. Int Tinnitus J 2009;15:161-3.

14) McNerney KM, Coad ML, Burkard R. The influence of caffeine on rotary chair and oculomotor testing. J Am Acad Audiol 2018;29:58795.

15) de Sousa AMA, Suzuki FA. Effect of caffeine on cervical vestibularevoked myogenic potential in healthy individuals. Braz J Otorhinolaryngol 2014;80:226-30.

16) Souza MEDCA, Costa KVTD, Menezes PL. Effect of caffeine on vestibular evoked myogenic potential: a systematic review with meta-analysis. Braz J Otorhinolaryngol 2018;84:381-8.

17) Litman RE, Hommer DW, Clem T, Rapaport MH, Pato CN, Pickar D. Smooth pursuit eye movements in schizophrenia: effects of neuroleptic treatment and caffeine. Psychopharmacol Bull 1989;25:473-8.

18) Pilli R, Naidu MUR, Pingali UR, Takallapally RKR. An electrooculographic method for the evaluation of psychotropic drugs on saccadic eye movements in healthy subjects. Int J Psychol Stud 2012;4: 75-87. 
19) Vlajkovic SM, Abi S, Wang CJ, Housley GD, Thorne PR. Differential distribution of adenosine receptors in rat cochlea. Cell Tissue Res 2007;328:461-71

20) Ricci A, Norris C, Guth P. Cyclic AMP modulates sensory-neural communication at the vestibular end organ. Brain Res 1991;565:78-84.

21) Seidl R, Peryl A, Nicham R, Hauser E. A taurine and caffeine-containing drink stimulates cognitive performance and well-being. Amino Acids 2000;19:635-42.

22) Kamimori GH, McLellan TM, Tate CM, Voss DM, Niro P, Lieberman HR. Caffeine improves reaction time, vigilance and logical reasoning during extended periods with restricted opportunities for sleep. Psychopharmacology (Berl) 2015;232:2031-42.

23) Santos VG, Santos VR, Felippe LJ, Almeida JW Jr, Bertuzzi R, Kiss MA, et al. Caffeine reduces reaction time and improves performance in simulated-contest of taekwondo. Nutrients 2014;6:637-49.
24) Dixit A, Vaney N, Tandin OP. Evaluation of cognitive brain functions in caffeine users: a P3 evoked potential study. Indian J Physiol Pharmacol 2006;50:175-80.

25) Deslandes AC, Veiga H, Cagy M, Piedade R, Pompeu F, Ribeiro P. Effects of caffeine on visual evoked potential (P300) and neuromotor performance. Arq Neuropsiquiatr 2004;62(2B):385-90.

26) Kawamura N, Maeda H, Nakamura J, Morita K, Nakazawa Y. Effects of caffeine on event-related potentials: comparison of oddball with single-tone paradigms. Psychiatry Clin Neurosci 1996;50:217-21.

27) Lorist MM, Tops M. Caffeine, fatigue, and cognition. Brain Cogn 2003;53:82-94.

28) Taghavi SMR, Geshani A, Rouhbakhsh N, Jalaie S, Pourbakht A, Kebriaeezadeh A, et al. Effects of short-term caffeine consumption on speech and sound reception in individuals with normal hearing. Aud Vestib Res 2018;27:150-6. 